\title{
GENETIC CONTROL OF RECOMBINATION IN SCHIZOPHYLLUM COMMUNE: THE OCCURRENCE AND SIGNIFICANCE OF NATURAL VARIATION
}

\author{
JUDITH STAMBERG* \\ Department of Genetics, The University, Birmingham, England and \\ Biological Laboratories, Harvard University, Cambridge, Mass., U.S.A.
}

Received 1.x.68

\section{INTRODUCTION}

That frequencies of recombination between linked genes are alterable by the genotype has been known almost since the discovery of linkage itself. Gowen (1919), who was the first to observe that recombination frequencies varied with the genetic background, concluded that "crossing over is one of the most highly variable phenomena known "-a conclusion whose validity has since been amply demonstrated. The literature now abounds with reports on the effects of genetic background on chiasma formation and genetic recombination.

Recent work, particularly with fungi, has shown that highly specific genes and gene systems exist for the control of recombination frequencies in limited regions of the genome. This has been termed the "fine control " of recombination (Stamberg, 1968; Simchen and Stamberg, 1969a). Four such fine-control genes have been found in $\mathcal{N}$ eurospora (Jessop and Catcheside, 1965; Smith, 1966; Catcheside, 1966; Jha, 1967). In Schizophyllum commune two independent gene systems control recombination between the component loci of the $A$ and $B$ incompatibility factors (Stamberg, 1968, 1969; Simchen and Connolly, 1968); gene systems have also been found that control frequencies of recombination in specific regions both linked and unlinked to the incompatibility factors (Simchen and Stamberg, 1969b). The effects of these systems are confined to limited segments of linkage groups; adjacent regions are, in at least some cases, controlled by different systems.

The existence of an extensive series of fine-control genes of recombination raises questions about their evolutionary significance. The present study is an attempt to assess the evolutionary value of two fine-control systems, by using dominance relationships and allelic distribution in nature as criteria.

\section{MATERIALS AND METHODS}

Strains 991, 14, 699 and E908 were obtained from the collection of wildtype Schizophyllum strains of Professor J. R. Raper. Their origins and mating types are:

\begin{tabular}{rll} 
Strain & Mating type & \multicolumn{1}{c}{ Origin } \\
991 & $A 97$ B97 & Costa Rica \\
14 & $A 4 B 4$ & Illinois \\
699 & $A 41 B 41$ & Massachusetts \\
E908 & $A 43 B 43$ & $\begin{array}{l}\text { Alabama, and } \\
\text { backcrossing programme } \\
\end{array}$ \\
& & to 699
\end{tabular}

* Present address: Laboratory of Genetics, The Hebrew University, Jerusalem, Israel. 
Explanation of the mating system of Schizophyllum and the use of the incompatibility factors as markers in recombination studies, as well as procedures for performing crosses and test matings, can be found in a previous publication (Stamberg, 1968). Cultures were maintained at $18^{\circ} \mathrm{C}$. prior to the collection of spores.

\section{Results}

1. Dominance relationships. The four strains were crossed in the six possible combinations, and frequencies of recombination in the $A$ factor and the $B$ factor were determined for each cross (table l). Recombination frequencies varied significantly from cross to cross. Five of the six crosses were homogeneous with respect to recombination in the $A$ factor, whereas the crosses fell into two groups of three each with respect to recombination in the $B$ factor.

TABLE 1

Natural variation in recombination frequencies in the $\mathrm{A}$ and $\mathrm{B}$ factors

\begin{tabular}{|c|c|c|c|c|c|c|}
\hline \multirow[b]{2}{*}{ Cross } & \multirow[b]{2}{*}{ Sample } & \multicolumn{2}{|c|}{ Rec. $A$} & \multirow[b]{2}{*}{ Sample } & \multicolumn{2}{|c|}{ Rec. $B$} \\
\hline & & No. & $\%$ & & No. & $\%$ \\
\hline 1. $991 \times 14 \dagger$ & 158 & 5 & $3 \cdot 2$ & 179 & 16 & $8 \cdot 9$ \\
\hline 2. $699 \times 14 \dagger$ & 551 & 21 & 3.8 & 363 & 23 & $6 \cdot 3$ \\
\hline 3. $991 \times 699$ & 157 & 7 & $4 \cdot 5$ & 157 & 12 & $7 \cdot 6$ \\
\hline 4. $14 \times \mathrm{E} 908$ & 174 & 9 & $5 \cdot 2$ & 174 & 5 & $2 \cdot 9$ \\
\hline 5. $991 \times$ E908 & 178 & 10 & $5 \cdot 6$ & 178 & 2 & $1 \cdot 1$ \\
\hline 6. $669 \times \mathrm{E} 908$ & 181 & 25 & $13 \cdot 8$ & 181 & 4 & $2 \cdot 2$ \\
\hline$\chi_{(5)}^{2}$ & & & $11 * * *$ & & & $5 * * *$ \\
\hline
\end{tabular}

Homogeneous grouping of crosses:

\section{A Factor}

Crosses 1, 2, 3, 4, 5: $\chi_{(4)}^{2}=1.91$

Pooled value $=4.3 \%$

Cross $6: 13 \cdot 8 \%$

\section{B Factor}

Crosses $4,5,6: \chi_{(2)}^{2}=1 \cdot 36$

Pooled value $=2 \cdot 1 \%$

Crosses 1, 2, 3: $\chi_{(2)}^{2}=1 \cdot 24$

Pooled value $=7 \cdot 3 \%$

Significance levels in this and the following tables are indicated as: unstarred $=\mathbf{P}$ greater than $0.05 ;^{*}=\mathrm{P}$ of $0.05-0.01 ; * *=\mathrm{P}$ of $0.01-0.001 ; * * *=\mathrm{P}$ less than 0.001 .

$\dagger$ Recombination frequencies in the $A$ factor and $B$ factor were tested in different samples.

The results indicate that, in both regions, low frequency of recombination is dominant to high. The reasoning behind this conclusion is as follows: if there are three strains such that $I \times 2$ gives high recombination, $I \times 3$ gives low recombination, and $2 \times 3$ gives low recombination, then strain 3 can be said to be carrying an allele (or alleles) for low recombination that is dominant to the allele (or alleles) for high recombination carried by strains 1 and 2 . For the $A$ factor, this relationship exists among the strains 699, E908, and 14, and also among the strains 699, E908, and 991 :

$699 \times$ E908: high $A$ factor recombination

$699 \times 14$ : low $A$ factor recombination

$\mathrm{E} 908 \times 14$ : low $A$ factor recombination 
Also:

$699 \times$ E908: high $A$ factor recombination

$699 \times 991$ : low $A$ factor recombination

$\mathrm{E} 908 \times 991$ : low $A$ factor recombination

Thus, strains 14 and 991 carry dominant alleles for low recombination in the $A$ factor, whereas strains 699 and E908 carry recessive alleles for high recombination in this region.

For the $B$ factor, a similar relationship exists among any two of the strains 991, 14, and 699, with E908 as the third strain:

\section{$991 \times 14 \quad$ : high $B$ factor recombination $991 \times \mathrm{E} 908$ : low $B$ factor recombination $14 \times \mathrm{E} 908$ : low $B$ factor recombination $991 \times 699$ : high $B$ factor recombination $991 \times$ E908: low $B$ factor recombination $699 \times \mathrm{E} 908$ : low $B$ factor recombination \\ $14 \times 699$ : high $B$ factor recombination $14 \times$ E908 : low $B$ factor recombination $699 \times$ E908: low $B$ factor recombination}

Strain E908, therefore, carries a dominant allele for low recombination in the $B$ factor, whereas strains 14,991, and 699 carry recessive alleles for high recombination in this region.

2. Distribution of alleles in nature. Surveys of recombination in the $A$ factor and in the $B$ factor, in a large number of wild-type strains taken from a worldwide sample, have been made by Raper and associates (Raper, Baxter and Ellingboe, 1960; Koltin, Raper and Simchen, 1967). The main purpose of these surveys was to establish the allelic constitution of the native $A$ factors and $B$ factors, but in addition they can be used to obtain information on the range and distribution of the recombination-control systems in nature.

(a) Control system for the A factor. Thirty-four wild-type strains were each crossed to a single common strain (699); the frequency of recombination in the $A$ factor was determined for each cross after meiosis at $33^{\circ} \mathrm{C}$. (Raper, Baxter and Ellingboe, 1960). (Actually thirty-eight crosses were made; four are excluded from the present analysis either because the meiotic temperature varied from $33^{\circ} \mathrm{C}$. or because the strains had a common allele and hence recombinants could not be detected.) The frequencies of recombination in the $A$ factor ranged from 3.3 to $22 \cdot 8$ per cent. and showed great heterogeneity. Our analysis of the data (table 2) shows that crosses 1-25 form a group within which all the variation is attributable simply to sampling error; similarly, crosses 17-34 form such a group. In other words, crosses 1-16 are unambiguously low and crosses 26-34 unambiguously high, while crosses 17-25 could belong to either group. This suggests that there are two separate groups whose distributions are partly overlapping, i.e. the variation among the crosses is explainable by an allelic difference in one gene. Strains 26-34 carry an allele for high frequency of recombination in the $A$ factor, whereas strains 1-16 carry an allele for low frequency of recombination. From the data it is not possible to tell which among strains 17-25 have the allele for high recombination frequency and which the allele for low. An alternative 
explanation is that additional allelic differences among the strains result in intermediate groups as well as high and low groups. On either explanation, however, the size of the unambiguously low group in comparison to that of

TABLE 2

A factor recombination in crosses involving a random sample of native strains ${ }^{1}$

\begin{tabular}{|c|c|c|c|c|c|c|}
\hline \multirow[b]{2}{*}{ Strain } & \multirow{2}{*}{$\begin{array}{l}\text { No. of cross } \\
\text { in original } \\
\text { paper }\end{array}$} & \multirow{2}{*}{$\begin{array}{l}\text { Parental } \\
A \text { factor }^{2}\end{array}$} & \multirow[b]{2}{*}{ Sample } & \multirow{2}{*}{$\begin{array}{c}\% \text { Rec. } \\
A\end{array}$} & \multicolumn{2}{|c|}{$\chi^{2}$ Significance level ${ }^{3}$} \\
\hline & & & & & low group & high group \\
\hline 1 & 27 & A35 & 123 & $3 \cdot 3$ & & $* * *$ \\
\hline 2 & 28 & $A 65$ & 121 & $3 \cdot 3$ & & $* * *$ \\
\hline 3 & 35 & $A 78$ & 78 & $3 \cdot 8$ & & $* * *$ \\
\hline 4 & 38 & A123 & 127 & $3 \cdot 9$ & & $* * *$ \\
\hline 5 & 12 & $A 1$ & 127 & 3.9 & & $* * *$ \\
\hline 6 & 5 & $A 42$ & 300 & $4 \cdot 7$ & & $* * *$ \\
\hline 7 & 30 & $A 69$ & 121 & $5 \cdot 0$ & & $* * *$ \\
\hline 8 & 31 & $A 72$ & 116 & $5 \cdot 2$ & & $* * *$ \\
\hline 9 & 37 & $A 88$ & 128 & $5 \cdot 5$ & & $* * *$ \\
\hline 10 & 20 & $A 99$ & 118 & $5 \cdot 9$ & & $* * *$ \\
\hline 11 & 33 & $A 74$ & 97 & $6 \cdot 2$ & & $* * *$ \\
\hline 12 & 13 & $A 71$ & 127 & $6 \cdot 3$ & & $* *$ \\
\hline 13 & 17 & $A 89$ & 124 & $7 \cdot 3$ & & $* *$ \\
\hline 14 & 7 & $A 2$ & 493 & $7 \cdot 3$ & & $* *$ \\
\hline 15 & 29 & $A 55$ & 102 & $7 \cdot 8$ & & $*$ \\
\hline 16 & 3 & $A 49$ & 127 & $7 \cdot 9$ & & * \\
\hline 17 & 21 & $A 102$ & 113 & $8 \cdot 0$ & & \\
\hline 18 & 25 & $A 124$ & 123 & $8 \cdot 1$ & & \\
\hline 19 & 14 & $A 79$ & 113 & $8 \cdot 8$ & & \\
\hline 20 & 15 & $A 86$ & 121 & $9 \cdot 1$ & & \\
\hline 21 & 4 & $A 47$ & 127 & $9 \cdot 4$ & & \\
\hline 22 & 23 & $A 116$ & 127 & $9 \cdot 4$ & & \\
\hline 23 & 32 & $A 73$ & 109 & $10 \cdot 1$ & & \\
\hline 24 & 24 & $A 120$ & 106 & $10 \cdot 4$ & & \\
\hline 25 & 8 & $A 45$ & 116 & $11 \cdot 2$ & & \\
\hline 26 & 2 & $A 51$ & 405 & $12 \cdot 3$ & * & \\
\hline 27 & 34 & $A 91$ & 120 & $12 \cdot 5$ & $*$ & \\
\hline 28 & 19 & $A 112$ & 108 & 13.0 & ** & \\
\hline 29 & 22 & $A 114$ & 117 & $13 \cdot 7$ & $* *$ & \\
\hline 30 & 10 & $A 21$ & 224 & 13.8 & $* * *$ & \\
\hline 31 & 18 & $A 92$ & 120 & $14 \cdot 2$ & $* * *$ & \\
\hline 32 & 6 & $A 43$ & 127 & $15 \cdot 7$ & $* * *$ & \\
\hline 33 & 16 & $A 87$ & 116 & $17 \cdot 2$ & $* * *$ & \\
\hline 34 & 36 & $A 118$ & 123 & $22 \cdot 8$ & $* * *$ & \\
\hline
\end{tabular}

1 Data from Raper, Baxter and Ellingboe (1960), table la.

2 The common mate was strain $699(A 41)$.

${ }^{3}$ Heterogeneity $\chi^{2}$ values were calculated for each possible sequential division into low and high groups. The significance levels for cross 7, for example, show that crosses 1-7 are homogeneous whereas crosses 7-34 are not. Thus, cross 7 belongs in the low group. See table 1 for explanation of asterisks.

the unambiguously high group suggests that alleles for low frequency of recombination are more widely distributed in nature than are those for high frequency of recombination. Of the 25 crosses in the two unambiguous groups, 16 (64 per cent.) carry an allele for low recombination.

(b) Control system for the B factor. Eighteen wild-type strains from the worldwide collection were each crossed to strain 699 ; the frequencies of recombination in the $B$ factor were determined for each cross at $30^{\circ} \mathrm{C}$. 
(Koltin, Raper and Simchen, 1967). Values were heterogeneous, ranging from $0 \cdot 1$ to $8 \cdot 0$ per cent. The crosses, with two exceptions, are divisible into unambiguous low and high groups (table 3 ). The variation can be attributed

TABLE 3

B factor recombination in crosses involving a random sample of native strains ${ }^{1}$

\begin{tabular}{|c|c|c|c|c|c|}
\hline \multirow[b]{2}{*}{ Strain } & \multirow[b]{2}{*}{$\begin{array}{l}\text { Parental } \\
B \text { factor }^{2}\end{array}$} & \multirow[b]{2}{*}{ Sample } & \multirow[b]{2}{*}{$\begin{array}{c}\% \text { Rec. } \\
B\end{array}$} & \multicolumn{2}{|c|}{$\chi^{2}$ Significance level ${ }^{3}$} \\
\hline & & & & low group & high group \\
\hline 1 & B79 & 692 & $0 \cdot 1$ & - & - \\
\hline 2 & $B 95$ & 468 & $0 \cdot 6$ & & $* * *$ \\
\hline 3 & $B 127$ & 379 & 0.8 & & $* * *$ \\
\hline 4 & $B 50$ & 568 & 0.9 & & $* * *$ \\
\hline 5 & $B 39$ & 314 & $1 \cdot 3$ & & $* * *$ \\
\hline 6 & $B 105$ & 305 & $1 \cdot 3$ & & $* * *$ \\
\hline 7 & $B 10$ & 298 & $1 \cdot 3$ & & $* * *$ \\
\hline 8 & $B 77$ & 580 & $1 \cdot 4$ & & $* * *$ \\
\hline 9 & B9 & 198 & $1 \cdot 5$ & & $* *$ \\
\hline 10 & $B 56$ & 243 & $1 \cdot 7$ & & $* *$ \\
\hline 11 & $B 65$ & 242 & $2 \cdot 1$ & & $* *$ \\
\hline 12 & $B 69$ & 300 & $2 \cdot 3$ & & $* *$ \\
\hline 13 & B53 & 392 & $2 \cdot 6$ & & $*$ \\
\hline 14 & $B 118$ & 366 & $3 \cdot 0$ & & \\
\hline 15 & $B 3$ & 371 & $4 \cdot 0$ & $*$ & \\
\hline 16 & $B 38$ & 308 & $4 \cdot 9$ & $* * *$ & \\
\hline 17 & $B 4$ & 174 & $6 \cdot 3$ & $* * *$ & \\
\hline 18 & $B 97$ & 200 & $8 \cdot 0$ & $* * *$ & \\
\hline
\end{tabular}

1 Data from Koltin, Raper and Simchen (1967), table 1.

2 The common mate was strain 699 (B4l).

$3 \chi^{2}$ values were calculated as in table 2, except that cross 1 was omitted from the calculations as it was found that there was no satisfactory division into two groups.

to a one-allele difference among the strains. Strains 15-18 carry an allele for high frequency of recombination; strains 2-13 have an allele for low frequency of recombination; strain 14 could belong to either group. The other exceptional cross (No. 1) has a recombination frequency significantly lower than that of crosses $2-13\left(\chi_{(1)}^{2}=7 \cdot 76^{* *}\right)$; this may be caused by a physical difference, e.g. a deletion between the loci of B79. Additional experiments would be required to test whether in fact variation in recombination in this one case has a structural rather than a genetic basis. Of the 16 crosses in the two unambiguous groups, 12 (75 per cent.) carry an allele for low recombination.

\section{Discussion}

The incompatibility factors are naturally occurring markers-i.e. extended series of alternate alleles at the $\alpha$ and $\beta$ loci of both factors exist in nature. They have the special function of controlling the breeding system. In a tetrapolar species such as Schizophyllum commune, the maximum potential of inbreeding-if no intrafactor recombination occurs-is 25 per cent. This value, which is the proportion of compatible matings that can occur among sibling progeny from one dikaryon, is increased, however, as the frequency of intrafactor recombination rises (Simchen, 1967). If the incompatibility factors consisted of only one locus each, the inbreeding potential 
would be 25 per cent., the minimum possible for a tetrapolar species. The outbreeding potential, on the other hand, is a function of the number of factor specificities (Raper, Krongelb and Baxter, 1958), and the presence of two loci, each with a series of alternate alleles, increases the number of factor specificities enormously. The presence of two-locus incompatibility factors plus genes controlling the amount of recombination between the pairs of linked loci appears to be a compromise between the needs to maximise outbreeding (by increasing the number of factor specificities) and minimise inbreeding (by keeping the frequency of intrafactor recombination low). This view is supported by two lines of evidence which indicate that low recombination within the incompatibility factors has a greater selective advantage to the species than does high recombination: (1) both control systems for recombination are structured in such a way that low frequency of recombination is dominant to high (previous indications that this was true for the $A$ factor were obtained by Simchen (1967) and by Simchen and Connolly (1968)) and (2) alleles for low recombination, in both control systems, are found more frequently in nature than are the alleles for high recombination. Simchen (1967) similarly concluded that low recombination would maintain high outbreeding and low inbreeding potentials and observed that high recombination would be advantageous "only under special circumstances such as isolation or colonisation of a new habitat".

The role of elements of the fine control of recombination in other regions of the genome is not obvious. Most of the regions for which genetic control of recombination has been demonstrated are delimited by markers for nutritional deficiencies that have been induced in the laboratory by mutagenic treatments. Nutritional deficiencies are rarely found in strains isolated from nature; presumably such mutations arise spontaneously but lead to such a decrease in fitness that they do not enter the gene pool in any significant measure. Hence, it is improbable that the fine-control genes of recombination that do exist in nature have as their main function the regulation of recombination between two such markers. To use a specific example, a gene system has been shown to control recombination, in Schizophyllum, between the markers nicotinic acid-2 and uracil-1 (Simchen and Stamberg, 1969b). In the laboratory, an altered frequency of recombination in this region leads to an altered frequency of prototrophs, so there is a conceivable advantage to the laboratory organism in having a fine control of recombination in this region. But in nature the loci nic-2 and ura-1 must exist, with vanishingly rare exceptions, only in competent condition; that is, they are capable of specifying the synthesis of functional enzymes. Recombination in this region between two chromosomes carrying functional nic-2 and ura-1 alleles will not lead to a better combination of these alleles. Hence, any advantage gained by controlling recombination in the region between nic-2 and ura-1 in nature is not likely to be directly related to these genes per se.

Genes that do "matter" in nature and which conceivably have evolved in such a way that recombination leading to their reassortment would be disadvantageous are genes collectively responsible for "fitness" characteristics such as growth rate, fruiting competence, spore production, and viability. The importance of linkage in keeping such groups of genes together was first recognised by Fisher (1930); Mather (1943) introduced the term " polygene" for a linked complex of genes that as a group promote fitness of the organism in some respect. He maintained that polygenes are a compromise between 
immediate fitness and potential variability, the frequency of recombination within the polygene controlling the rate of release of the variability. "Consequently the recombination frequency is itself an adaptive character and will be subject to selection."

It is in this light that the fine-control genes of recombination should be considered. Fine controls that regulate recombination specifically between the genes of a polygenic complex would control the rate of release of variability stored within that complex and thus would be advantageous to the species. Other genes, such as those involved in the synthesis of metabolites, that happen to be located among the genes of a fitness complex would be regulated by the same fine control as the genes of that complex. Single fitness genes are usually difficult to map and use as markers for genetic experiments. It is this fact that leads to the somewhat ironical situation in which, it is proposed, elements of the fine control of recombination are important in keeping certain combinations of genes together, but because of the limitations of our experimental methods we observe only a byproduct of this function, i.e. the control of other genes fortuitously located nearby.

Alternatively, loci such as nic-2 and ura-1 that are involved in the synthesis of metabolites may themselves be part of polygenic complexes; that is, natural selection may have caused the grouping of " modifier" genes, that collectively improve the functioning of the gene products of the nic-2 and/or ura-1 loci, around the latter loci. The fine-control genes responsible for recombination in the nic-2-ura-1 region would then have as their primary function the maintenance of the "major" genes and the "modifying" loci as an intact unit. The incompatibility factors with their fine control systems are the only cases where it is highly likely that natural fitness complexes and their controls are being directly observed.

The recombination frequency that provides the species with the optimal rate of release of variability could well be different for different fitness complexes. Thus, separate recombination-control genes would be necessary in order to maintain the different optimal levels simultaneously.

\section{Summary}

1. Low frequencies of recombination between the component loci of the $A$ and $B$ incompatibility factors of Schizophyllum commune are dominant to high frequencies.

2. Alleles for low recombination in both incompatibility factors are found more frequently in nature than are the alleles for high recombination.

3. Low recombination within the incompatibility factors seems, therefore, to be of selective advantage to the species. The advantage of low recombination in these regions is probably that the inbreeding potential is minimised while the two-locus structure of the incompatibility factors is maintained to maximise the outbreeding potential.

4. It is suggested that the numerous and complex components of the finecontrol that regulates recombination in Schizophyllum are important in maintaining combinations of genes that, as groups, promote fitness of the organism, and in releasing variation within such combinations at rates optimal for the species. 
Acknowledgments.--The author would like to thank Professor J. L. Jinks and Dr G. Simchen for generously providing laboratory facilities, for valuable discussions and suggestions concerning the analysis of data, and for critically reading the manuscript. The investigation was supported by a Public Health Service fellowship from the U.S. National Institutes of Health. Financial support during the preparation of the manuscript was kindly made available by Professor J. R. Raper from NIH grant AI 06124.

\section{REFERENGES}

CATcheside, D. G. 1966. A second gene controlling allelic recombination in Neurospora crassa. Australian 7. Biol. Sci., 19, 1039-1046.

FISHER, R. A. 1930. The Genetical Theory of Natural Selection. Clarendon Press, Oxford.

GOWEN, J. W. 1919. A biometrical study of crossing over. On the mechanism of crossing over in the third chromosome of Drosophila melanogaster. Genetics, 4, 205-250.

JEssop, A. P., AND CATcheside, D. G. 1965. Interallelic recombination at the his-1 locus in Neurospora crassa and its genetic control. Heredity, 20, 237-256.

JHA, K. K. 1967. Genetic control of allelic recombination at the histidine-3 locus of Neurospora crassa. Genetics, 57, 865-873.

KOLTIN, Y., RAPER, J. R., AND SIMCHEN, G. 1967. The genetic structure of the incompatibility factors of Schizophyllum commune: the $B$ factor. Proc. Nat. Acad. Sci. (Wash.), 57, 55-62.

Mather, K. 1943. Polygenic inheritance and natural selection. Biol. Revs. Camb. Phil. Soc., $18,32-64$.

RAPER, J. R., BAXTER, M. G., AND ELLINGBOE, A. H. 1960. The genetic structure of the incompatibility factors in Schizophyllum commune: the A-factor. Proc. Nat. Acad. Sci. (Wash.), $46,833-842$.

RAPER, J. R., KRONGELB, G. S., AND BAXTER, M. G. 1958. The number and distribution of incompatibility factors in Schizophyllum. Amer. Nat., 92, $221-232$.

SIMCHEN, G. 1967. Genetic control of recombination and the incompatibility system in Schizophyllum commune. Genet. Res., Camb., 9, 195-210.

SIMCHEN, G., AND CONNOLLY, v. 1968. Changes in recombination frequency following inbreeding in Schizophyllum. Genetics, 58, 319-326.

SIMCHEN, G., AND STAMBERG, J. 1969a. Fine and coarse controls of genetic recombination. Nature (Lond.), 222, 329-332.

SIMCHEN, G., AND STAMBERG, J. 1969b. Genetic control of recombination in Schizophyllum commune. Specific and independent regulation of adjacent and non-adjacent chromosomal regions. Heredity, 24, 369-381.

sMith, B. R. 1966. Genetic controls of recombination. 1. The recombination-2 gene of Neurospora crassa. Heredity, 21, 481-498.

StAmberG, J. 1968. Two independent gene systems controlling recombination in Schizophyllum commune. Molec. Gen. Genetics, 102, 221-228.

STAMBERG, J. 1969. Genetic control of recombination in Schizophyllum commune: Separation of the controlled and controlling loci. Heredity, 24, 306-309. 